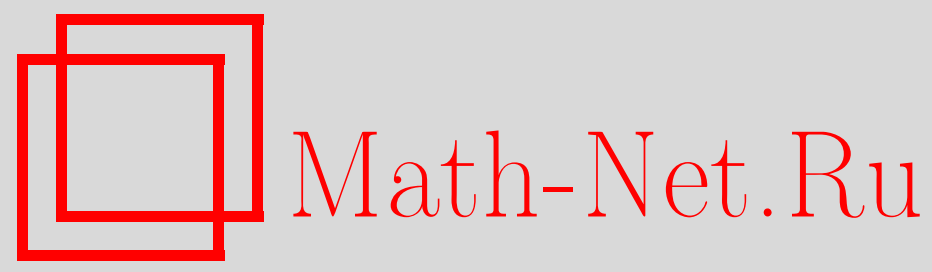

А. И. Герко, Асимптотически рекуррентные решения уравнений в $\beta$-производных, Матем. заметки, 2000, том 67, выпуск $6,837-851$

DOI: https://doi.org/10.4213/mzm902

Использование Общероссийского математического портала Math-Net.Ru подразумевает, что вы прочитали и согласны с пользовательским соглашением http://www.mathnet.ru/rus/agreement

Параметры загрузки:

IP : 54.209 .52 .79

26 апреля 2023 г., 16:56:59

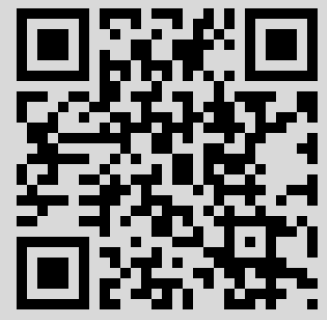




\section{АСИМПТОТИЧЕСКИ РЕКУРРЕНТНЫЕ РЕШЕНИЯ УРАВНЕНИЙ В $\beta$-ПРОИЗВОДНЫХ}

\section{А. И. Герко}

В статье методами теории расширений динамических систем изучаются уравнения в $\beta$-производных со свойствами единственности решения и непрерывной зависимости решений от начальных данных и правых частей уравнения. На уравнения в $\beta$-производных (в частности, в полных производных) распространяются теоремы ЖиковаБронштейна об асимптотически почти периодических решениях обыкновенного дифференциального уравнения. Кроме асимптотической почти периодичности рассмотрены асимптотическая рекуррентность, слабая асимптотическая дистальность и асимптотическая дистальность. С уравнениями связываются динамические системы, которые порождаются пространством правых частей и пространствами решений и начальных значений решений уравнения. Фазовые полугруппы динамических систем, вообще говоря, не локально компактны.

Библиографияя: 27 названий.

Введение. В статье исследуются дифференциальные уравнения

$$
y^{\prime}=f(x, y),
$$

где независимая переменная $x$ принимает значения из некоторого подмножества отделимого вещественного топологического векторного пространства $E$; неизвестная функция $y$ принимает значения из некоторого подмножества отделимого полного локально выпуклого вещественного или комплексного топологического векторного пространства $T$; отображение $f$ определено на подмножестве из $E \times T$ и принимает значения в пространстве линейных непрерьвных отображений $E \rightarrow T$; штрих означает $\beta$-производную [1] (в частности, производную $\Phi$ реше), где $\beta$ - некоторая совокупность ограниченных подмножеств из $E$.

В монографиях [2], [3] имеется обширная библиография, посвященная различным частным случаям уравнений (1) и их применениям. Наиболее известным и изученньп представителем таких уравнений является обыкновенное дифференциальное уравнение в банаховом пространстве. К уравнениям (1) сводятся системы дифференциальных уравнений $x_{t_{j}}^{\prime}=\varphi_{j}(t, x)\left(j=1,2, \ldots, m, t \in \mathbb{R}^{m}, x(t) \in \mathbb{R}^{n}\right)$ с частными производными. В [3] отмечается, что одним из интересных и важных классов уравнений, которые сводятся $\mathrm{K}$ уравнениям (1) в бесконечномерных пространствах, являются уравнения в вариационных (функциональных) производных (например, уравнение Шрёдингера [4], уравнение 
Швингера [5]). Уравнения (1) широко применяются в различных разделах теоретической физики, дифференциальной геометрии, теории групा Ли, электродинамике, оптимальном управлении системами с распределенными параметрами, теории стохастических систем с импульсными воздействиями и др.

Теория уравнений (1) сохранила глубокие аналогии с теорией обыкновенных дифференциальных уравнений. Общие вопросы теории изложены, в частности, в монографиях [2] и [3] (в [2] пространства $E$ и $T$ конечномерны, в [3] рассмотрены линейные уравнения). В этих монографиях а также в статьях [6]-[12] рассмотрены и некоторые аспекты качественной теории уравнений (1) (как правило, для конечномерногопространства $E$ ).

Целью настоящей статьи является распространение на уравнения в $\beta$-производных известной теоремы Жикова-Бронштейна-Черний о положительной асимптотической почти периодичности компактного равномерно асимптотически устойчивого решения обыкновенного дифференциального уравнения с почти периодической правой частью (см., например, [13]-[16]). Кроме асимптотической почти периодичности мы рассматриваем асимптотическую рекуррентность, слабую асимптотическую дистальность и асимптотическую дистальность.

Обычно асимптотически почти периодической называют функцию, представимую в виде суммы почти периодической функции и бесконечно малой на бесконечности функции. Поэтому при исследовании многомерных уравнений возникает необходимость интерпретировать понятие предела на бесконечности. В монографии [2], в частности, при исследовании автономных динамических систем это делается с помошью сходимости по фильтру. Отметим, что в одномерном случае понятие предела на бесконечности связано с упорядочением области определения решений отношением ">", определяемым так: $x>y \Longleftrightarrow x=y+z$ для некоторого положительного числа $z$. Непосредственное обобщение этого определения, основанное на использовании операции “+”, предопределяет возможность применять теорию расширений обших динамических систем (полугрупп преобразований) для исследования неавтономных многомерных уравнений. Использование же сходимости по фильтру не позволяет непосредственно применять к исследованиям теорию расширений полугрупп преобразований. В наших исследованиях мы пользуемся как непосредственным обобщением отношения ">" через операцию "+" на области определения решений, так и некоторым абстрактным отношением $\rho$ на области определения решений. Используя различные обобщения предела на бесконечности и учитьвая некоторые другие факторы, мы предлагаем различные виды определений асимптотического поведения решений уравнения. Из полученных нами теорем, в частности, при соответствующей конкретизации отношения $\rho$ (например, $(a, b) \in \rho \Longleftrightarrow\|a\|>\|b\|)$ следуют результаты, используюшие сходимость по фильтру (по фильтру Немьщкого).

По аналогии с обыкновенными дифференциальными уравнениями мы связываем с уравнениями некоторые динамические системы в пространствах правых частей, решений и начальных значений решений уравнения. При этом, поскольку в качестве "пространства-времени" у нас выступает, вообще говоря, бесконечномерное пространство, мы используем динамические системы с, вообе говоря, не локально компактными фазовыми полугруппами; функциональные пространства мы наделяем равномерной структурой равномерной сходимости на элементах некоторого абстрактного семейства подмножеств из области определения рассматриваемых отображений (в отличие от общепринятой равномерной структуры компактной сходимости). 
Наши определения в идейном плане практически не отличаются от общепринятых в случае одномерной области определения функций. Однако, ввиду большого выбора различных "направлений" и конусов в многомерном "пространстве-времени" мы учитываем возможность применения их к исследованию свойств функций при изменении аргументов в определенном "направлении", в частности, к исследованию свойств функций (решений уравнений) по части аргументов. В качестве таких "направлений" могут выступать некоторые полугрупшы (конусы) из области определения функций (наполненные полугрупшы, полугруппы, порождающие группу).

Основные соглашения. Всюду в статье мы используем следующие обозначения и соглашения.

$E$ - отделимое вещественное топологическое векторное пространство.

$T$ - отделимое полное локально вьпуклое вещественное или комплексное топологическое векторное пространство.

$P \subset E, S$ - подполугруппа групшы $E, 0 \in S \subset P$ и $S+P \subset P ; W$ - отделимое подмножество из $T$.

$\beta, \tau(P), \tau(W)$ и $\tau(S)$ - некоторые совокупности подмножеств из $E, P, W$ и $S$ соответсвенно, для которых вьполнены условия:

1) $\beta$ - некоторая совокупность ограниченных подмножеств, содержащая все одноточечные подмножества из $E$;

2) каждая точка из $S$ имеет окрестность, принадлежащую $\tau(S)$;

3 ) объединение любого конечного семейства множеств из $\tau(S)$ (соответственно из $\tau(P), \tau(W))$ принадлежит $\tau(S)$ (соответственно $\tau(P), \tau(W))$;

4) система $\tau(S)$ (соответственно $\tau(P), \tau(W)$ ) покрывает $S$ (соответственно $P, W$ );

5) $\forall A \in \tau(S) \forall B \in \tau(P) \exists C \in \tau(P) A+B \subset C$.

$Z$ - векторное пространство линейных непрерьвных отображений $E \rightarrow T$ с $\beta$-топологией.

$\Phi_{1}$ - множество равномерно непрерывных на каждом множестве из $\tau(P) \times \tau(W)$ отображений $P \times W \rightarrow Z$, снабженное равномерной структурой $\tau(P) \times \tau(W)$-сходимости.

$\sigma_{1}$ - отображение $\Phi_{1} \times S \rightarrow Z^{P \times W}$, определяемое по правилу $\sigma_{1}(f, s)=f_{s}$, где $f_{s}(p, t)=f(s+p, t)((p, t) \in P \times W)$.

$\Phi_{2}-$ множество равномерно непрерывных на каждом множестве из $\tau(P)$ отображений $P \rightarrow T$, снабженное равномерной структурой $\tau(P)$-сходимости.

$\sigma_{2}$ - отображение $\Phi_{2} \times S \rightarrow T^{P}$, определяемое по правилу $\sigma_{2}(f, s)=f_{s}$, где $f_{s}(p)=$ $f(s+p)(p \in P)$.

$\mathscr{U}[X]$ - равномерность пространства $X$, согласованная с топологией на $X$.

В работе мы широко пользуемся терминологией, символикой и результатами теории топологических групп и полугрупп преобразований. Для большей полноты изложения приведем некоторые определения (подробнее см., например, [15]-[18]).

Под полугруппой преобразований мы понимаем тройку $(X, S, \pi)$, где $X$ - топологическое пространство и $\pi: X \times S \rightarrow X$-непрерьвное отображение такое, что для любых $x \in X, s, t \in S \pi(x, 0)=x$ и $\pi(\pi(x, s), t)=\pi(x, s+t)$.

При сделанных вьше предположениях можно доказать, что $\left(\Phi_{i}, S, \sigma_{i}\right)$ - полугруппа преобразований - абстрактный аналог динамической системы сдвигов Бебутова $(i=$ $1,2)$. 
Для полугруппы преобразований $(X, S, \pi), D \subset S$ и $x \in X$ символом $x D$ обозначаем множество $\{\pi(x, s) \mid s \in D\}$.

Расширением $\varphi:(X, S, \pi) \rightarrow(Y, S, \rho)$ полугрупп преобразований $(X, S, \pi)$ и $(Y, S, \rho)$ назьваем непрерьвную сюръекцию $\varphi: X \rightarrow Y$, для которой $\varphi(\pi(x, s))=\rho(\varphi(x), s)$ $(x \in X, s \in S)$.

Пусть $\varphi:(X, S, \pi) \rightarrow(Y, S, \rho)$ - расширение полугрупп преобразований; $x \in X$; $M \subset X ; D, Q \subset S$ - непустые подмножества; $[D]$ - некоторьй класс непустых подмножеств множества $D$.

Множество $M$ назьвается [D]-устойчивым типа $S_{1}$ относительно расиирения $\varphi[18]$, если для любого $\alpha \in \mathscr{U}[X]$ существуют $A \in[D]$ и $\beta \in \mathscr{U}[X]$, для которых $\left((M \times X) \cap R_{\varphi} \cap \beta\right) A \subset \alpha$.

Множество $M$ назьвается $[D]$-устойчивым типа $S_{2}$ относительно расиирения $\varphi[18]$, если существует окружение $\beta \in \mathscr{U}[X]$ и для любого $\alpha \in \mathscr{U}[X]$ существуют $A \in[D]$ и $p \in D$ такие, что $\left((M \times X) \cap R_{\varphi} \cap \beta\right) p A \subset \alpha$.

Точка $x$ называется $[D] Q$-асимптотически поточечно почти периодической относительно расширения $\varphi$ [18], если существует компактное минимальное множество $M \subset \overline{x Q}$ такое, что для некоторой точки $y \in M \cap \varphi^{-1}(\varphi(x))$ и произвольного окружения $\alpha \in \mathscr{U}[X]$ сушествует пара $(q, A) \in Q \times[D]$, для которой $(x, y) q A \subset \alpha$.

Точка $x$ называется $[D] Q$-асимптотически слабо дистальной относительно расширения $\varphi$ [18], если существует компактное множество $M \subset \overline{x Q}$ такое, что для некоторой дистальной в $M$ точки $y \in M \cap \varphi^{-1}(\varphi(x))$ и произвольного окружения $\alpha \in \mathscr{U}[X]$ существует пара $(q, A) \in Q \times[D]$, для которой $(x, y) q A \subset \alpha$.

Точка $x$ назьвается $[D] Q$-асимптотически дистальной $([D] Q$-асимптотически регионально дистальной) относительно расиирения $\varphi$ [18], если существует компактное дистальное (регионально дистальное) множество $M \subset \overline{x Q}$ такое, что для некоторой точки $y \in M \cap \varphi^{-1}(\varphi(x))$ и произвольного окружения $\alpha \in \mathscr{U}[X]$ существует пара $(q, A) \in Q \times[D]$, для которой $(x, y) q A \subset \alpha$.

Если $[D]=\{D\}$, то в приведенных определениях символ $[D]$ заменяем на $D$.

Подножество $D$ из $S$ называется наполненным, если для произвольного компакта $K \subset S$ найдется элемент $p \in D$ такой, что $p+K \subset D$.

Уравнения в $\beta$-производных и связанные с ними расширения полугрупп преобразований. В этом пункте мы уточняем объект исследования - уравнения в $\beta$-производных с памятью. Кроме этого мы указьваем некоторые расширения, к исследованию которых по существу сводится исследование уравнений.

ОПРЕДЕЛЕНИЕ 1. $\beta$-производной отображсения $\varphi: P \rightarrow T$ в точке $x$ назьвается элемент $\varphi^{\prime}(x) \in Z$ такой, что для любых $A \in \beta, \eta \in \mathscr{U}[T]$ существует число $\delta>0$ такое, что для всех $\tau \in \mathbb{R}, 0<|\tau|<\delta$, и всех $a \in A$ вьполняется соотношение

$$
\left(\tau^{-1}(\varphi(x+\tau a)-\varphi(x)), \varphi^{\prime}(x) a\right) \in \eta
$$

ОПРЕДЕЛЕНИЕ 2. Уравнением в $\beta$-производных относительно функиии у называем уравнение $y^{\prime}(x)=f(x, y(x))$, где $f$ - отображение $P \times W \rightarrow Z$ и $y^{\prime}(x)$ означает $\beta$-производную функции $y$ в точке $x$. Уравнение в $\beta$-производных назьваем уравнением в полных производных, если $\beta$ - совокупность всех ограниченных подмножеств из $E$. 
Обычно уравнение $y^{\prime}(x)=f(x, y(x))$ записываем короче в форме $y^{\prime}=f(x, y)$, т.е. в форме (1).

В дальнейшем считаем, что "правые части" $f$ уравнений $(1)$ принадлежат $\Phi_{1}$, решения уравнения (1) принадлежат $\Phi_{2}$, и уравнения в полных производных рассматриваем при следующих предположениях: $E$ - квазиполное нормированное пространство, при этом $P=E$, если пространство $E$ бесконечномерно, и $P$ - вьпуклое множество, если пространство $E$ конечномерно; $\tau(P)$ (соответственно $\tau(W), \tau(S)$ ) - совокупность всех компактных подмножеств из $P$ (соответственно из $W, S)$.

Справедливы следующие две леммы замкнутости для уравнений (1).

ЛЕмма 1. Пусть $K \in \tau(W), I$ - направленное мноэсество и выполнены условия:

1) любая $\beta$-липиичева кривая, соединяющая произвольные две точки из $P$, содер ится в некотором множсесте, принадлежащем $\tau(P)$;

2) каждая ломаная в $P$ является $\beta$-липшичевой (это выполняется, в частности, если $\beta$ содержит все конечномерные ограниченные множества uз $E)$;

3) для любого $i \in I$ функиия $\varphi_{i}$ - решение уравнения $y^{\prime}=f_{i}(x, y)$ в $\beta$-производных, причем $\overline{\varphi_{i}(P)} \subset K$

4) $\lim _{i} f_{i}=f \quad$ в $\Phi_{1} u \lim _{i} \varphi_{i}=\varphi$ в $\Phi_{2}$.

Тогда $\varphi$-решение уравнения $y^{\prime}=f(x, y)$ в $\beta$-производных и $\overline{\varphi(P)} \subset K$.

ДокАЗАтЕльСтво. Для $g \in \Phi_{1}$ и $\psi \in \Phi_{2}$ символом $g^{\psi}$ обозначаем отображение, определяемое правилом $g^{\psi}(x)=g(x, \psi(x))(x \in P)$. Из условия леммы следует, что направленность $\left\{f_{i}^{\varphi_{i}}\right\}$ сходится к функции $f^{\varphi}$ равномерно на множествах из $\tau(P)$.

Пусть $x_{0}, x_{1} \in P$ и $\Gamma-\beta$-липшицева кривая в $P$, соединяющая точки $x_{0}$ и $x_{1}$. Поскольку $f_{i}\left(x, \varphi_{i}(x)\right)=\varphi_{i}^{\prime}(x)$ для любого $x \in P$, то по теореме 3.6 из [1]

$$
\int_{\Gamma} \varphi_{i}^{\prime}(x) d x=\varphi_{i}\left(x_{1}\right)-\varphi_{i}\left(x_{0}\right), \quad i \in I .
$$

По теореме 3.1 из [1]

$$
\begin{aligned}
\int_{\Gamma} f(x, \varphi(x)) d x & =\lim _{i} \int_{\Gamma} f_{i}\left(x, \varphi_{i}(x)\right) d x=\lim _{i} \int_{\Gamma} \varphi_{i}^{\prime}(x) d x \\
& =\lim _{i}\left(\varphi_{i}\left(x_{1}\right)-\varphi_{i}\left(x_{0}\right)\right)=\varphi\left(x_{1}\right)-\varphi\left(x_{0}\right),
\end{aligned}
$$

т.е. $\int_{\Gamma} f(x, \varphi(x)) d x=\varphi\left(x_{1}\right)-\varphi\left(x_{0}\right)$. Мы получили, что интеграл $\int_{\Gamma} f(x, \varphi(x)) d x$ зависит только от начальной и конечной точек пути интегрирования. В таком случае по теореме 3.7 из [1] для любого $t \in P$ и фиксированного $x_{0} \in P$

$$
\varphi^{\prime}(t)=\left(\varphi(t)-\varphi\left(x_{0}\right)\right)^{\prime}=\left(\int_{x_{0}}^{t} f(x, \varphi(x)) d x\right)^{\prime}=f(t, \varphi(t)),
$$

т.е. $\varphi^{\prime}(t)=f(t, \varphi(t))$. Понятно, что $\overline{\varphi(P)} \subset K$.

Следующую лемму мы докажем для уравнений в полных производных. Предварительно отметим следующее. 
Пусть $E$ - нормированное пространство, $\beta$ - совокупность всех ограниченных подмножеств из $E$ и $\widetilde{Q}$ - семейство непрерьвных полунорм на $T$, порождающих топологию на $T$. Если $A \in Z$ и $q \in \widetilde{Q}$, то согласно предложению 1.1 из [19, с. 96] существует число $c>0$ такое, что $q(A x) \leqslant c\|x\|$ для любого $x \in E$. Для произвольного $q \in \widetilde{Q}$ через $p_{q}$ обозначаем отображение $Z \rightarrow \mathbb{R}$, определяемое правилом

$$
p_{q}(A)=\inf \{c \mid c>0, q(A x) \leqslant c\|x\|(x \in E)\} .
$$

Непосредственно проверяется, что $p_{q}$ - полунорма на $Z$,

$$
p_{q}(A)=\sup _{\|x\| \leqslant 1} q(A x) \quad \forall A \in Z
$$

и семейство полунорм $Q^{*}=\left\{p_{q} \mid q \in \widetilde{Q}\right\}$ определяет $\beta$-топологию на $Z$.

ЛЕмма 2. Пусть $K \in[W]$ и для любого $i \in I$ функиия $\varphi_{i}$ - решение уравнения $y^{\prime}=f_{i}(x, y)$ в полных производных, причем $\overline{\varphi_{i}(P)} \subset K u \lim _{i} f_{i}=f$ в $\Phi_{1}$. Тогда

1) семейство $\overline{\left\{\varphi_{i} \mid i \in I\right\}}$ компактно;

2) предел $\varphi$ произвольной поднаправленности направленности $\left\{\varphi_{i}\right\}$ является решением уравнения $y^{\prime}=f(x, y)$ в полных производных и $\overline{\varphi(P)} \subset K$.

ДокАЗАтЕЛьство. Через $\mathscr{U}[T]$ обозначим равномерность пространства $T$, порождающую топологию на $T$ и инвариантную относительно сдвигов. Последнее означает, что для произвольного базиса $\mathcal{N}$ окрестностей нуля совокупность $\mathscr{U}^{*}[T]=\left\{\alpha_{V} \mid V \in \mathscr{N}\right\}$ множеств $\alpha_{V}=\{(x, y) \mid x, y \in T, x-y \in V\}$ является базисом для $\mathscr{U}[T]$.

В силу теоремы Асколи [20, с. 308] для доказательства компактности множества $\overline{\left\{\varphi_{i} \mid i \in I\right\}}$ достаточно доказать равностепенную непрерывность множества $\left\{\varphi_{i} \mid i \in I\right\}$ в каждой точке произвольного компакта из $P$. Пусть $A$ - компакт из $P, A^{\prime}-$ замкнутая выпуклая оболочка $A$. Тогда $A^{\prime}$ - компакт из $P$. Предположим, что $t_{1} \in A$ и $\alpha-$ произвольное окружение базы $\mathscr{U}^{*}[T]$ равномерности $\mathscr{U}[T]$. Существуют натуральные числа $n, m$ и непрерывные полунормы $q_{k} \in \widetilde{Q}(k=1,2, \ldots, m)$ такие, что $(x, y) \in \alpha$ тогда и только тогда, когда $q_{k}(x-y) \leqslant n^{-1}$ для произвольного $k=1,2, \ldots, m$. Обозначим

$$
M_{i}^{k}=\sup _{z \in A^{\prime} \times K} p_{q_{k}}\left(f_{i}(z)\right), \quad i \in I, \quad k=1,2, \ldots, m .
$$

Тогда $M_{i}^{k}=p_{q_{k}}\left(f_{i}\left(z_{i}^{k}\right)\right)$ для некоторого $z_{i}^{k} \in A^{\prime} \times K(i \in I, k=1,2, \ldots, m)$. Считаем, что для произвольного $k=1,2, \ldots, m \lim _{i} z_{i}^{k}=z^{k} \in A^{\prime} \times K$. Докажем, что $\lim _{i} f_{i}\left(z_{i}^{k}\right)=f\left(z^{k}\right)$ для произвольного $k=1,2, \ldots, m$. Так как $\lim _{i} z_{i}^{k}=z^{k}$ и отображение $f$ непрерьвно на множестве $A^{\prime} \times K$, то для натуральных чисел $p, l$ и непрерьвных полунорм $p_{r} \in Q^{*}(r=1,2, \ldots, l)$ существует $i_{1} \in I$ такое, что для произвольных $i>i_{1}$, $k=1,2, \ldots, m$ и $r=1,2, \ldots, l$

$$
p_{r}\left(f\left(z_{i}^{k}\right)-f\left(z^{k}\right)\right) \leqslant(2 p)^{-1} .
$$

Поскольку $\lim _{i} f_{i}=f$, то существует $i_{2} \in I$ такое, что для произвольных $i>i_{2}$, $k=1,2, \ldots, m$ и $r=1,2, \ldots, l$

$$
p_{r}\left(f_{i}\left(z_{i}^{k}\right)-f\left(z_{i}^{k}\right)\right) \leqslant(2 p)^{-1} .
$$


С учетом (2) и (3) получим для произвольных $i>\max \left(i_{1}, i_{2}\right), k=1,2, \ldots, m$ и $r=1,2, \ldots, l$ оценку

$$
p_{r}\left(f_{i}\left(z_{i}^{k}\right)-f\left(z^{k}\right)\right) \leqslant p_{r}\left(f_{i}\left(z_{i}^{k}\right)-f\left(z_{i}^{k}\right)\right)+p_{r}\left(f\left(z_{i}^{k}\right)-f\left(z^{k}\right)\right) \leqslant p^{-1},
$$

т.е. $p_{r}\left(f_{i}\left(z_{i}^{k}\right)-f\left(z^{k}\right)\right) \leqslant p^{-1}$. Доказанное означает, что $\lim _{i} f_{i}\left(z_{i}^{k}\right)=f\left(z^{k}\right)$ для произвольного $k=1,2, \ldots, m$. В силу непрерывности полунорм $p_{q_{k}}$ из доказанного следует, что

$$
\lim _{i} p_{q_{k}}\left(f_{i}\left(z_{i}^{k}\right)\right)=p_{q_{k}}\left(f\left(z^{k}\right)\right)
$$

для произвольного $k=1,2, \ldots, m$. Поэтому для произвольного $k=1,2, \ldots, m$ существует

$$
\sup _{i \in I} M_{i}^{k}=M^{k} \in \mathbb{R} .
$$

Предположим, что $M>\max _{k} M^{k}$ и $t_{2} \in A$ таково, что $\left\|t_{1}-t_{2}\right\|<(n M)^{-1}$. Тогда, учитьвая теорему о среднем, для произвольного $k=1,2, \ldots, m$ получим оценку

$$
\begin{aligned}
q_{k}\left(\varphi_{i}\left(t_{1}\right)-\varphi_{i}\left(t_{2}\right)\right) & \leqslant \sup _{t \in\left[t_{1}, t_{2}\right]} q_{k}\left(\varphi_{i}^{\prime}(t)\left(t_{2}-t_{1}\right)\right) \leqslant \sup _{t \in\left[t_{1}, t_{2}\right]} p_{q_{k}}\left(\varphi_{i}^{\prime}(t)\right)\left\|t_{2}-t_{1}\right\| \\
& <\sup _{t \in\left[t_{1}, t_{2}\right]} p_{q_{k}}\left(f_{i}\left(t, \varphi_{i}(t)\right)\right)(n M)^{-1} \leqslant M \cdot(n M)^{-1}=n^{-1},
\end{aligned}
$$

т.е. $q_{k}\left(\varphi_{i}\left(t_{1}\right)-\varphi_{i}\left(t_{2}\right)\right)<n^{-1}$. Это означает, что $\left(\varphi_{i}\left(t_{1}\right), \varphi_{i}\left(t_{2}\right)\right) \in \alpha$ для произвольного $i \in I$. Мы доказали равностепенную непрерывность множества $\left\{\varphi_{i} \mid i \in I\right\}$ в точке $t_{1}$. Утверждение 1) леммы доказано. Утверждение 2) леммы следует из леммы 1 с учетом того, что $\beta$-топология на $Z$ порождается семейством полунорм $Q^{*}$.

ОПРЕДЕлЕниЕ 3. Решение $\varphi$ уравнения (1) называется компактным, если $\overline{\varphi(P)} \subset W$ и множество $\overline{\varphi(P)}$ компактно. Решение $\varphi$ уравнения $(1)$ назьвается $\tau(W)$-поглощаемым, если $\overline{\varphi S} \subset \Phi_{2}$ и $\overline{\varphi(P)} \subset K$ для некоторого $K \in \tau(W)$.

Понятно, что каждое компактное решение уравнения в полных производных является $\tau(W)$-поглощаемым. Понятно также, что для компактности множества $\overline{\varphi S}$ достаточно выполнения одного из следующих условий:

1) отображение $\varphi$ равномерно непрерьвно;

2) множество $f(P, \overline{\varphi(P)})$ ограничено;

3) множество $\overline{f S}$ компактно.

Всюду в дальнейшем мы (аналогично одномерному случаю) будем рассматривать уравнения со свойствами единственности решения и непрерьвной зависимости решений от начальных данных и правых частей уравнения. Такие уравнения мы будем назьвать уравнениями с памятью. Приведем формальные определения.

ОПРЕДЕЛЕНИЕ 4. Уравнение (1) в $\beta$-производных называем уравнением $с$ памятью $W$, если выполнены условия:

1) для любой пары $(s, \omega) \in P \times W$ сушествует решение $\varphi$ уравнения $(1)$ такое, что $\varphi(s)=\omega ;$

$2)$ если для решений $\varphi$ и $\psi$ уравнения (1) вьполняется равенство $\varphi(s)=\psi(s)$ для некоторого $s \in P$, то $\varphi=\psi$; 
$3)$ для произвольного решения $\varphi$ уравнения (1), произвольных окружения $\alpha \in \mathscr{U}[W]$ и множества $K \in \tau(P)$ найдется окружение $\beta \in \mathscr{U}[W]$ такое, что если для некоторого решения $\psi$ уравнения $(1)(\varphi(0), \psi(0)) \in \beta$, то $(\varphi(k), \psi(k)) \in \alpha$ для любого $k \in K$;

4) для $f$, произвольньх $\alpha \in \mathscr{U}[W], K \in \tau(P)$ и $M \in \tau(W)$ существует $\beta \in \mathscr{U}[Z]$ такое, что для произвольного решения $\varphi$ уравнения (1) и произвольного решения $\psi$ уравнения $y^{\prime}=g(x, y)$ в $\beta$-производных из соотношений $\varphi(0)=\psi(0)$ и $(f(k, m), g(k, m)) \in \beta$ для любой пары $(k, m) \in K \times M$ следует соотношение $(\varphi(k), \psi(k)) \in \alpha$ для любого $k \in K$.

Через $\mathscr{A}$ обозначаем класс уравнений $y^{\prime}=f(x, y)$ с памятью $W$, для которых из $g \in \overline{f S}$ следует, что $y^{\prime}=g(x, y)$ - уравнение с памятью $W$, и выполнены условия:

1) для любого $A \in \tau(S)$ существует $B \in \tau(P) A \subset B$;

2 ) любая $\beta$-липшицева кривая, соединяющая произвольные две точки из $P$, содержится в некотором множестве, принадлежащем $\tau(P)$;

$3)$ каждая ломаная в $P$ является $\beta$-липшицевой.

Считаем в дальнейшем, что уравнение (1) принадлежит классу $\mathscr{A}$.

Пусть $\varphi-\tau(W)$-поглощаемое решение уравнения (1) (в частности, компактное решение уравнения (1) в полных производных); $\pi: \overline{f S} \times W \times S \rightarrow \overline{f S} \times W$ - отображение, задаваемое по правилу $\pi(g, w, s)=\left(g_{s}, \psi(s)\right)$, где $\psi$ - решение уравнения $y^{\prime}=g(x, y)$, для которого $\psi(0)=w ; X=\left\{(g, \psi) \mid g \in \overline{f S}, \psi\right.$ - решение уравнения $\left.y^{\prime}=g(x, y)\right\}$; $\mathscr{P}: \overline{f S} \times W \rightarrow \overline{f S}$ и $\mathscr{P}^{*}: X \rightarrow \overline{f S}$ - проектирования; $\lambda: X \rightarrow \overline{f S} \times W$ - отображение, задаваемое по правилу

$$
\lambda(g, \psi)=(g, \psi(0)) .
$$

Понятно, что $X \subset \Phi_{1} \times \Phi_{2}-$ инвариантное множество в прямом произведении $\left(\Phi_{1} \times \Phi_{2}\right.$, $\left.S, \sigma_{1} \times \sigma_{2}\right)$ полугрупп преобразований $\left(\Phi_{1}, S, \sigma_{1}\right)$ и $\left(\Phi_{2}, S, \sigma_{2}\right)$. Поэтому определена полугруппа преобразований $\left(X, S, \sigma_{12}\right)$ - подполугруппа преобразований полугруппы преобразований $\left(\Phi_{1} \times \Phi_{2}, S, \sigma_{1} \times \sigma_{2}\right)$. Кроме этого, используя стандартные рассуждения, можно доказать следующую лемму.

Лемма 3. Справедливы следующие утверәсдения.

1) $(\overline{f S} \times W, S, \pi)$ - полугруппа преобразований, изоморфная полугруппе преобразований $\left(X, S, \sigma_{12}\right)$ при изоморфизме $\lambda$, определяемом правилом (4).

2) Проектирования $\mathscr{P}$ и $\mathscr{P}^{*}$ определяют расширения $\mathscr{P}:(\overline{f S} \times W, S, \pi) \rightarrow(\overline{f S}$, $\left.S, \sigma_{1}\right)$ и $\mathscr{P}^{*}:\left(X, S, \sigma_{12}\right) \rightarrow\left(\overline{f S}, S, \sigma_{1}\right)$ полугрупп преобразований.

3) Определены две полугруппы преобразований: $\left(\overline{(f, \varphi) S}, S, \sigma_{12}\right)$ - подполугруппа полугруппы преобразований $\left(X, S, \sigma_{12}\right)$ u $(\overline{(f, \varphi(0)) S}, S, \pi)$ - подполугруппа полугруппы преобразований $(\overline{f S} \times W, S, \pi)$. Эти полугруппы преобразований изоморфны при изоморфизме $\lambda^{*}$, где $\lambda^{*}$ - сужсние отображсения $\lambda$ по формуле (4) на множество $\overline{(f, \varphi) S}$.

Основные результаты. Охарактеризуем основные классы функций, с которыми будем в дальнейшем работать.

ОПРЕДЕЛЕНИЕ 5. Пусть $Q \subset S$ и $f \in \Phi_{1}$. 
Функция $f$ называется $S Q$-рекуррентной в смысле Биркгофа, если для любых $A \in$ $\tau(P), C \in \tau(W)$ и $\alpha \in \mathscr{U}[Z]$ сушествует компакт $K$ из $Q$ такой, что для любого $s \in Q$ существует $t \in K$, для которого $(f(a, c), f(s+t+a, c)) \in \alpha$ при произвольных $(a, c) \in A \times C$.

Функция $f$ назьвается слабо $S Q$-дистальной, если для любого $g \in \overline{f S}, f \neq g$, существуют $A \in \tau(P), C \in \tau(W)$ и $\alpha \in \mathscr{U}[Z]$ такие, что для любого $s \in Q$ существует $(a, c) \in A \times C$, для которой $(f(s+a, c), g(s+a, c)) \notin \alpha$.

Функция $f$ называется $S Q$-дистальной, если для любых $g, h \in \overline{f S}, g \neq h$, существуют $A \in \tau(P), C \in \tau(W)$ и $\alpha \in \mathscr{U}[Z]$ такие, что для любого $s \in Q$ существует $(a, c) \in A \times C$, для которой $(g(s+a, c), h(s+a, c)) \notin \alpha$.

Функция $f$ называется $S Q$-почти периодической в смысле Бора, если для любых $A \in \tau(P), C \in \tau(W)$ и $\alpha \in \mathscr{U}[Z]$ существует компакт $K \subset Q$ такой, что для произвольного $t \in Q$ существует $k \in K$, для которого $(f(s+a, c), f(s+t+k+a, c)) \in \alpha$ при любых $(s, a, c) \in S \times A \times C$.

Приведенные определения естественно распространяются на функции из $\Phi_{2}$ и функции-пары из $\Phi_{1} \times \Phi_{2}$.

Понятно, что функция $f \in \Phi_{i}(i=1,2)$ является $S Q$-рекуррентной в смысле Биркгофа (слабо $S Q$-дистальной, $S Q$-дистальной, $S Q$-почти периодической в смысле Боpa) тогда и только тогда, когда точка $f Q$-почти периодична (точка $f Q$-дистальна, множество $\overline{f S} Q$-дистально, множество $\overline{f S} Q$-почти периодично) в полугрушпе преобразований $\left(\overline{f S}, S, \sigma_{i}\right)[17],[18]$. Поэтому наши определения по сушеству удовлетворяют конщепщиям применения методов топологической динамики к исследованию конкретных классов уравнений [15], [16], и для них справедливы свойства, отмеченные в [21].

$S Q$-рекуррентность функции в смысле Биркгофа и $S Q$-почти периодичность функции в смысле Бора можно охарактеризовать в терминах "относительной плотности множества $\varepsilon$-периодов" [2] функции. Пусть для $f \in \Phi_{2}, M \in \tau(P)$ и $\alpha \in \mathscr{U}[T]$

$$
A_{Q, M, \alpha}^{f}=\{q \mid q \in Q,(f(m), f(q+m)) \in \alpha(m \in M)\} .
$$

Непосредственно из определений следует, что функция $f \in \Phi_{2} S Q$-рекуррентна в смысле Биркгофа тогда и только тогда, когда для любых $M \in \tau(P), \alpha \in \mathscr{U}[T]$ существует компакт $K \subset Q$ такой, что $Q \subset A_{Q, M, \alpha}^{f}-K$ и $0 \in A_{Q, M, \alpha}^{f}$. Аналогично, функция $f \in \Phi_{2} S Q$-почти периодична в смысле Бора тогда и только тогда, когда для любых $M \in \tau(P), \alpha \in \mathscr{U}[T]$ существует компакт $K \subset Q$ такой, что

$$
Q \subset \bigcap_{s \in S} A_{Q, M, \alpha}^{f_{s}}-K, \quad 0 \in \bigcap_{s \in S} A_{Q, M, \alpha}^{f_{s}} .
$$

При $Q=S=P=E$ и $\tau(P)=\{P\}$ (поскольку $\bigcap_{s \in E} A_{E, E, \alpha}^{f_{s}}=A_{E, E, \alpha}^{f}$ ) мы получаем, что функция $f E E$-почти периодична в смысле Бора тогда и только тогда, когда для любого $\alpha \in \mathscr{U}[T]$ существует компакт $K \subset E$ такой, что $E=A_{E, E, \alpha}^{f}+K$. Это хорошо согласуется с определением почти периодической функции из [2] (ибо в конечномерном случае для $E E$-почти периодической в смысле Бора функции $f$ множество $\overline{f E}$ компактно, и в равенстве $E=A_{E, E, \alpha}^{f}+K$ множество $K$ можно считать конечным).

Отметим, что наиболее полно изучены почти периодические функции (см., например, [22]). О рекуррентных и дистальных функциях см., например, в [23]-[26]. 
Следующие определения являются обобщениями на многомерньй случай понятий равномерно устойчивого в положительном направлении, положительно притягивающего и положительно асимптотически почти периодического (устойчивого по Пуассону) решения из [15], [16], [27].

ОПРЕДЕЛЕНИЕ 6 . Пусть $D, Q \subset S,[D]$ - некоторая непустая совокупность подмножеств из $D$ и $\varphi$ - решение уравнения (1).

Решение $\varphi$ называется $[D] Q$-устойчивы.м, если для любого $\varepsilon \in \mathscr{U}[W]$ существуют $\delta \in \mathscr{U}[W]$ и $A \in[D]$ такие, что для любого решения $\psi$ уравнения (1) и любого $d \in Q$ из условия $(\varphi(d), \psi(d)) \in \delta$ следует для произвольного $a \in A$ соотношение $(\varphi(d+a), \psi(d+a)) \in \varepsilon$.

Решение $\varphi$ называется $[D] Q$-притягивающим, если существует $\delta \in \mathscr{U}[W]$ такое, что для любого $\varepsilon \in \mathscr{U}[W]$ существует пара $(A, d) \in[D] \times D$ такая, что для любого решения $\psi$ уравнения (1) и любого $p \in Q$ из условия $(\varphi(p), \psi(p)) \in \delta$ следует соотношение $(\varphi(d+p+a), \psi(d+p+a)) \in \varepsilon$ для любого $a \in A$.

Решение $\varphi$ назьвается $[D] Q$-асимптотически рекуррентным $([D] Q$-асимптотически слабо дистальным, $[D] Q$-асимптотически дистальным, $[D] Q$-асимптотически почти периодическим), если во множестве $\overline{\varphi Q}$ существует $S S$-рекуррентное в смысле Биркгофа (слабо $S S$-дистальное, $S S$-дистальное, $S S$-почти периодическое в смысле Бора) решение $\psi$ уравнения (1) такое, что для любого $\eta \in \mathscr{U}[W]$ существует пара $(A, d) \in[D] \times Q$, для которой при всех $t \in d+A$ вьполняется соотношение $(\varphi(t), \psi(t)) \in \eta$.

Если $[D]=\{D\}$, то в приведенных определениях квадратные скобки опускаем.

Отметим, что в наших определениях обобщение предела на бесконечности реализовано с помощью операции "+". Непосредственно, пользуясь определениями, можно доказать следуюшие две леммы, из которых следует, что наши определения $[D] Q$-устойчивого, $[D] Q$-притягивающего, $[D] Q$-асимптотически рекуррентного, $[D] Q$-асимптотически слабо дистального, $[D] Q$-асимптотически дистального и $[D] Q$-асимптотически почти периодического решения также удовлетворяют концепциям применения методов топологической динамики к исследованию конкретных классов уравнений.

Лемма 4. Решение ч уравнения (1) является $[D] Q$-устойчивым $([D] Q$-притягивающим тогда и только тогда, когда множество $(f, \varphi(0)) Q \quad[D]$-устойчиво типа $S_{1}\left(S_{2}\right)$ относительно расширения Я્Р из леммы 3.

ЛЕмма 5. Пусть $\varphi-\tau(W)$-поглощаемое решение уравнения (1). Если точка $(f, \varphi(0))[D] Q$-асимптотически поточечно почти периодична $([D] Q$-асимптотически слабо дистальна, $[D] Q$-асимптотически дистальна, $[D] Q$-асимптотически регионально дистальна) относительно расиирения Я из леммы 3 , то решение $\varphi$ $[D] Q$-асимптотически рекуррентно ([D]Q-асимптотически слабо дистально, $[D] Q$-асимптотически дистально, $[D] Q$-асимптотически почти периодично).

Tеорема 1. Пусть $Q$ - наполненная подполугруппа из $S, 0 \in Q, D \subset S ;$ функиия $f$ SQ-рекуррентна в смысле Биркгофа; $\varphi-\tau(W)$-поглощаемое решение уравнения (1) и множество $\overline{(f, \varphi(0)) Q}$ компактно. Тогда

1) если решение $\varphi[D] Q$-устойчиво, то оно $[D] Q$-асимптотически рекуррентно; 
2) Если $0 \in D$ и решение $\varphi[D] Q$-притягивающее, то оно $[D](Q+D)$-асимптотически рекуррентно.

ДокАЗАтЕльСтво. Если решение $\varphi[D] Q$-устойчиво, то по лемме 4 множество $(f$, $\varphi(0)) Q[D]$-устойчиво типа $S_{1}$ относительно расширения $\mathscr{P}$. Согласно теореме 5 из [18] точка $(f, \varphi(0))[D] Q$-асимптотически поточечно почти периодична относительно расширения $\mathscr{P}$. Поэтому по лемме 5 решение $\varphi[D] Q$-асимптотически рекуррентно. Аналогично доказывается вторая часть теоремы.

Tеорема 2. Пусть $S$ - группа, $D$ u $Q$ - наполненные подполугруппь из $S$, $L \subset S, 0 \in D \cap Q \cap L ;$ функиия $f$ слабо SQ-дистальна (SQ-дистальна); $\varphi$ $\tau(W)$-поглощаемое решение уравнения (1) и множество $\overline{(f, \varphi(0)) Q}$ компактно. Тогда справедливы следующие утверждения:

1) если решение $\varphi$ DQ-устойчиво, то оно $D Q$-асимптотически слабо дистально (DQ-асимптотически дистально);

2) если решение $\varphi$ DQ-устойчиво и $[L] Q$-притягивающее, то оно $[L](L+Q)$ асимптотически слабо дистально $([L](L+Q)$-асимптотически дистальHO).

ДокАЗАтЕльство. Доказательство следует схеме доказательства теоремы 1 с использованием теорем 6 и 7 из [18].

TеОрема 3. Пусть $S$ - группа, $D$ u $Q$ - наполненные подполугруппы из $S$, причем полугруппа $D$ такова, что для некоторого компакта $K$ из $D$ всякая открыто-замкнутая подполугруппа из $D$, содержащая $K$, совпадает $c D ; L \subset S$, $0 \in D \cap Q \cap L ; \varphi-\tau(W)$-поглощаемое решение уравнения (1). Если

1) мнохество $\overline{(f, \varphi(0)) Q}$ компактно,

2) функиия $f S Q$-почти периодична в смысле Бора,

3) решение $\varphi$ DQ-устойчиво и $[L] Q$-притягивающее,

то решение $\varphi$ DQ-асимптотически почти периодично и $[L](L+Q)$-асимптотически почти периодично.

ДокАЗАТЕЛЬСтво. Из условия теоремы следует, что $\overline{f S}$ - компактноеминимальное почти периодическое множество. Поэтому множество $\overline{f S}$ дистально. Согласно лемме 4 и утверждению 2 из [18] множество $\overline{(f, \varphi(0)) Q} D$-устойчиво типа $S_{1}$ и $[L]$-устойчиво типа $S_{2}$ относительно расширения $\mathscr{P}$. В силу компактности множество $\overline{(f, \varphi(0)) Q}$ содержит некоторое $Q$-минимальное и, следовательно, минимальное подмножество $M$. Докажем дистальность расширения $\left.\mathscr{P}\right|_{M}:(M, S) \rightarrow(\overline{f S}, S)$ (здесь $\left.\mathscr{P}\right|_{M}$ - сужение отображения $\mathscr{P}$ на множество $M)$. В силу теоремы 1 из [18] достаточно доказать дистальность расширения $\left.\mathscr{P}\right|_{M}:\left(M, D^{-1}\right) \rightarrow\left(\overline{f S}, D^{-1}\right)$. Пусть $y \in \overline{f S}$ и точки $x$ и $z$ из $M$ таковы, что $\mathscr{P}(x)=\mathscr{P}(z)=y$. Предположим, что точки $x$ и $z D^{-1}$-проксимальны; $\alpha \in \mathscr{U}[\overline{f S} \times W]-$ произвольное окружение. И пусть $\beta$ - окружение, соответствующее окружению $\alpha$ в силу $D$-устойчивости типа $S_{1}$ относительно расширения $\mathscr{P}$ множества $\overline{(f, \varphi(0)) Q}$. В силу $D^{-1}$-проксимальности точек $x$ и $z$ найдется элемент $p \in D$ такой, что $\left(x p^{-1}, z p^{-1}\right) \in \beta$. В таком случае $(x, z) \in \alpha$. В силу произвольности окружения $\alpha$ из $(x, z) \in \alpha$ следует, что $x=z$. Мы доказали дистальность слоя расширения $\left.\mathscr{P}\right|_{M}$ над точкой $y$. В 
силу произвольности точки $y$ и дистальности множества $\overline{f S}$ расширение $\left.\mathscr{P}\right|_{M}$ дистально. В таком случае отображение $\left.\mathscr{P}\right|_{M}$ открыто. Из $[L]$-устойчивости типа $S_{2}$ множества $\overline{(f, \varphi(0)) Q}$ относительно расширения $\mathscr{P}$ следует локальная $L$-проксимальность [18] каждого слоя расширения $\left.\mathscr{P}\right|_{M}$. Согласно теореме 4 из [18] слои расширения $\left.\mathscr{P}\right|_{M}$ конечны. Мы находимся в условиях теоремы 3 из [18]. Значит, множество $M$ регионально дистально, следовательно, почти периодично. Согласно лемме 1 из [18] во множестве $M$ существует точка $(f, \omega), Q$-проксимальная точке $(f, \varphi(0))$. Можно считать, что $\omega=\psi(0)$ для некоторого решения $\psi$ уравнения (1). Заметим, что согласно лемме 3 решение $\psi S S$-почти периодично в смысле Бора. Пусть $\alpha \in \mathscr{U}[\overline{f S} \times W]-$ произвольное окружение и $\beta \in \mathscr{U}[\overline{f S} \times W]$ - окружение, соответствующее $\alpha$ в силу $D$-устойчивости множества $\overline{(f, \varphi(0)) Q}$ типа $S_{1}$ относительно расширения $\mathscr{P}$. Так как точка $(f, \varphi(0))$ $Q$-проксимальна точке $(f, \psi(0))$, то $((f, \varphi(0)),(f, \psi(0))) d \in \beta$ для некоторого элемента $d \in Q$. В таком случае $((f, \varphi(0)),(f, \psi(0)))(d+D) \subset \alpha$, значит точка $(f, \varphi(0))$ $D Q$-регионально дистальна относительно расширения $\mathscr{P}$. Согласно лемме 5 решение $\varphi$ является $D Q$-асимптотически почти периодическим.

Докажем $[L](L+Q)$-асимптотическую почти периодичность решения $\varphi$. В силу $[L]$ устойчивости типа $S_{2}$ множества $\overline{(f, \varphi(0)) Q}$ относительно расширения $\mathscr{P}$ существует окружение $\beta \in \mathscr{U}[\overline{f S} \times W]$ такое, что для произвольного окружения $\alpha \in \mathscr{U}[\overline{f S} \times W]$ существует пара $(A, d) \in[L] \times Q$, для которой

$$
\left((\overline{(f, \varphi(0)) Q} \times \overline{f S} \times W) \cap R_{\mathscr{P}} \cap \beta\right)(d+A) \subset \alpha .
$$

Из $Q$-проксимальности точек $(f, \varphi(0))$ и $(f, \psi(0))$ следует, что $((f, \varphi(0)),(f, \psi(0))) p \in \beta$ для некоторого элемента $p \in Q$. В таком случае $((f, \varphi(0)),(f, \psi(0)))(p+d+A) \subset \alpha$ в силу соотношения $(5)$, значит точка $(f, \varphi(0))[L](L+Q)$-регионально дистальна относительно расширения $\mathscr{P}$. Согласно лемме 5 решение $\varphi[L](L+Q)$-асимптотически почти периодично.

Нижеследующие определения идейно ничем не отличаются от определений 6, однако в них реализована конщепия обобщения предела на бесконечности через некоторое абстрактное отношение на $S$.

ОПРЕДЕЛЕНИЕ 7. Пусть $D, Q \subset S$; $[D]$ - некоторая непустая совокупность подмножеств из $D ; \rho$ - некоторое отношение на $S ; \varphi$ - решение уравнения (1).

Решение $\varphi$ назьвается $\rho[D] Q$-yстойчивым, если для любого $\varepsilon \in \mathscr{U}[W]$ существуют $\delta \in \mathscr{U}[W]$ и $A \in[D]$ такие, что для произвольного решения $\psi$ уравнения (1) и произвольного $d \in Q$ из условия $(\varphi(d), \psi(d)) \in \delta$ следует для любого $a \in A,(a, d) \in \rho$, соотношение $(\varphi(a), \psi(a)) \in \varepsilon$.

Решение $\varphi$ назьвается $\rho[D] Q$-притягивающим, если существует $\delta \in \mathscr{U}[W]$ такое, что для произвольного $\varepsilon \in \mathscr{U}[W]$ существует пара $(A, p) \in[D] \times D$ такая, что для любого решения $\psi$ уравнения (1) и любого $d \in Q$ из условия $(\varphi(d), \psi(d)) \in \delta$ следует, что $(\varphi(a), \psi(a)) \in \varepsilon$ для всех $a \in A,(a, p+d) \in \rho$.

Решение $\varphi$ называется $\rho[D] Q$-асимптотически рекуррентным $(\rho[D] Q$-асимптотически слабо дистальным, $\rho[D] Q$-асимптотически дистальным. $\rho[D] Q$-асимптотически почти периодическим), если во множестве $\overline{\varphi Q}$ существует $S S$-рекуррентное в смысле Биркгофа (слабо $S S$-дистальное, $S S$-дистальное, $S S$-почти периодическое в смысле Бора) решение $\psi$ уравнения (1) такое, что для любого $\varepsilon \in \mathscr{U}[W]$ существует 
пара $(A, d) \in[D] \times Q$, для которой при всех $a \in A,(a, d) \in \rho$, выполняется соотношение $(\varphi(a), \psi(a)) \in \varepsilon$.

Если $[D]=\{D\}$, то в приведенных определениях квадратные скобки опускаем.

При некоторых ограничениях на $S, \rho, D$ и $Q$ можно доказьвать аналоги теорем $1-3$. В этих доказательствах символом $V(\alpha, \delta)$ мы обозначаем элемент базы равномерности $\mathscr{U}[\overline{f S} \times W]$, определяемьй правилом $V(\alpha, \delta)=\{((a, b),(m, n)) \mid(a, m) \in \alpha,(b, n) \in \beta\}$ (здесь $\alpha \in \mathscr{U}[\overline{f S}], \delta \in \mathscr{U}[W])$.

TеОрема 4. Пусть $Q$ - наполненная подполугруппа из $S, 0 \in Q, D \subset S$; функиия $f$ SQ-рекуррентна в смысле Биркгофа, $\varphi-\tau(W)$-поглощаемое решение уравнения (1) и мнохество $\overline{(f, \varphi(0)) Q}$ компактно. Тогда справедливы следующие утверэдения:

1) если решение $\varphi \rho[D] Q$-устойчиво, то оно $\rho[D] Q$-асимптотически рекуррентно;

2) если $0 \in D$ и решение $\varphi \rho[D] Q$-притягивающее, то оно $\rho[D](Q+D)$-асимптотически рекуррентно.

ДоКАЗАТЕЛЬСТво. Пусть решение $\varphi \rho[D] Q$-устойчиво. В условиях теоремы множество $\overline{f S}$ компактно и минимально. Во множестве $\overline{(f, \varphi(0)) Q}$ найдется $Q$-минимальное, следовательно, минимальное множество $M$. Согласно лемме 1 из [18] существует точка $(f, w) \in M, Q$-проксимальная точке $(f, \varphi(0))$. Считаем решение $\psi$ уравнения (1) таковым, что $w=\psi(0)$. Пусть $\varepsilon \in \mathscr{U}[W]$ и $\alpha \in \mathscr{U}[\overline{f S}]$ произвольны, а $\delta \in \mathscr{U}[W]$ и $A \in[D]$ соответствуют $\varepsilon$ в силу $\rho[D] Q$-устойчивости решения $\varphi$. В силу $Q$-проксимальности точек $(f, \varphi(0))$ и $(f, \psi(0))$ существует $d \in Q$ такое, что $\left(\left(f_{d}, \varphi(d)\right),\left(f_{d}, \psi(d)\right)\right) \in V(\alpha, \delta)$. Поэтому $(\varphi(d), \psi(d)) \in \delta$ и, следовательно, $(\varphi(a), \psi(a)) \in \varepsilon$ для всех $a \in A,(a, d) \in \rho$. Поскольку множества $\overline{(f, \varphi) Q}$ и $\overline{(f, \varphi(0)) Q}$ изоморфны, а точка $(f, \psi(0))$ принадлежит компактному минимальному множеству, то и точка $(f, \psi)$ принадлежит компактному минимальному множеству. В таком случае функция $\psi S S$-рекуррентна в смысле Биркгофа. Поскольку, очевидно, $\psi \in \overline{\varphi Q}$, то утверждение 1$)$ теоремы доказано. Аналогично доказывается утверждение 2) теоремы.

Tеорема 5. Пусть $S$ - группа, $D u Q$-наполненные подполугруппы из $S, L \subset S$, $0 \in Q \cap L, Q \subset D$; отношение $\rho$ таково, что для всех $d \in D, l \in Q \quad(d+l, l) \in \rho$; функиия $f$ слабо $S Q$-дистальна $(S Q$-дистальна); $\varphi-\tau(W)$-поглощаемое решение уравнения (1) имножество $\overline{(f, \varphi(0)) Q}$ компактно. Тогда справедливы следующие утверэдения:

1) если решение $\varphi$ рDQ-устойчиво, то оно $\rho D Q$-асимптотически слабо дистально ( $\rho D Q$-асимптотически дистально);

2) если решение ч $\rho D Q$-устойчиво и $\rho[L] Q$-притягивающее, то оно $\rho[L](L+Q)$ асимптотически слабо дистально $(\rho[L](L+Q)$-асимптотически дистальHO).

ДокАЗАТЕЛЬСтво. Из условия теоремы следует $D Q$-устойчивость решения $\varphi$. Кроме этого, множество $\overline{f S}$ компактно, минимально и точка $f$ дистальна в $\overline{f S}$ (и множество $\overline{f S}$ дистально). Как и в доказательстве теоремы 4 , во множестве $\overline{(f, \varphi(0)) Q}$ найдем компактное минимальное множество $M$, а во множестве $M$ найдем точку $(f, \psi(0))$, 
$Q$-проксимальную точке $(f, \varphi(0))$. Кроме этого, точка $(f, \psi(0))$ дистальна во множестве $M$ (множество $M$ дистально) и $\psi$-решение уравнения (1). В таком случае решение $\psi$ слабо $S S$-дистально ( $S S$-дистально) и $\psi \in \overline{\varphi Q}$. Пусть $\varepsilon \in \mathscr{U}[W]$ и $\alpha \in \mathscr{U}[\overline{f S}]$ произвольны, а $\delta \in \mathscr{U}[W]$ соответствует $\varepsilon$ в силу $\rho D Q$-устойчивости решения $\varphi$. В силу $Q$-проксимальности точек $(f, \varphi(0))$ и $(f, \psi(0))$ существует элемент $d \in Q$, для которого $\left(\left(f_{d}, \varphi(d)\right),\left(f_{d}, \psi(d)\right)\right) \in V(\alpha, \delta)$. Поэтому $(\varphi(d), \psi(d)) \in \delta$ и для всех $a \in D,(a, d) \in \rho$, выполняется соотношение $(\varphi(a), \psi(a)) \in \varepsilon$.

Tеорема 6. Пусть $S$ - группа, $D$ u $Q$ - наполненные подполугруппы из $S, n p u-$ чем полугруппа $D$ такова, что для некоторого компакта $K$ из $D$ всякая открыто-замкнутая подполугруппа из $D$, содержащая $K$, совпадает $c D ; L \subset S$, $0 \in D \cap Q \cap L ; Q \cup L \subset D$; отношение $\rho$ таково, что для всех $d \in D, p \in Q, l \in L$ $(d+p, p) \in \rho u(d+l, l) \in \rho ; \varphi-\tau(W)$-поглощаемое решение уравнения (1). Кроме того, пусть выполнены условия:

1) мнохсество $\overline{(f, \varphi(0)) Q}$ компактно;

2) функиия $f$ SQ-почти периодична в смысле Бора;

3) решение $\varphi ~ \rho D Q$-устойчиво и $\rho[L] Q$-притягивающее.

тогда решение ч $\rho D Q-и \rho[L](L+Q)$-асимптотически почти периодично.

ДоКАЗАТЕЛЬСтво. Из условия теоремы следует, что решение $\varphi D Q$-устойчиво и множество $\overline{f S}$ компактно, минимально и почти периодично. Как и в доказательстве теоремы 3 , во множестве $\bar{\varphi} Q$ найдется $S S$-почти периодическое в смысле Бора решение $\psi$ уравнения (1) такое, что точки $(f, \varphi(0))$ и $(f, \psi(0)) \quad Q$-проксимальны. Пусть $\varepsilon \in \mathscr{U}[W]$ и $\alpha \in \mathscr{U}[\overline{f S}]$ произвольны и $\delta \in \mathscr{U}[W]$ соответствует $\varepsilon$ в силу $\rho D Q$-устойчивости решения $\varphi$. В силу $Q$-проксимальности точек $(f, \varphi(0))$ и $(f, \psi(0))$ существует $d \in Q$ такое, что $\left(\left(f_{d}, \varphi(d)\right),\left(f_{d}, \psi(d)\right)\right) \in V(\alpha, \delta)$. Поэтому из условия $(\varphi(d), \psi(d)) \in \delta$ следует соотношение $(\varphi(a), \psi(a)) \in \varepsilon$ для всех $a \in A,(a, d) \in \rho$. Значит, решение $\varphi$ $\rho D Q$-асимптотически почти периодично. Аналогично доказываем $\rho[L](L+Q)$-асимптотическую почти периодичность решения $\varphi$.

ЗАмечаниЕ 1. Теоремы 1-6 верны, если условие $\tau(W)$-поглощаемости решения $\varphi$ заменить условием: $\varphi$ - компактное решение уравнения (1) в полных производных. При этом вместо компактности множества $\overline{(f, \varphi(0)) Q}$ достаточно потребовать компактность множества $\overline{f Q}$.

ЗАмЕчАниЕ 2. Теоремы $1-6$ и их аналоги согласно замечанию 1 для уравнений в полных производных верны, если полугруппы $Q$ и $D$ топологически порождают группу $S$. Топологическую порождаемость множеством групшы мы понимаем в следующем смысле. Пусть $S$ - топологическая группа, $L^{*}=L \cup(-L)$ и $M$ - множество всевозможных конечных сумм элементов из $L^{*}$. Если $S=\bar{M}$, то говорим, что множество $L$ топологически порождает групп $S$ (символом $-L$ обозначаем множество $\{p \mid-p \in L\}$ ).

\section{СПИСОК ЦИТИРОВАННОЙ ЛИТЕРАТУРЫ}

[1] Авербух В.И., Смолянов О.Г. Теория дифференцирования в линейных топологических пространствах // УМН. 1967. Т. 22. №6(138). С. 201-260.

[2] Гайшун И. В. Вполне разрешимые многомерные дифференциальные уравнения. Минск: Наука и техника, 1983.

[3] Гайшун И. В. Линейные уравнения в полных производных. Минск: Наука и техника, 1989. 
[4] Боголюбов Н. Н., Ширков Д. В. Введение в теорию квантованных полей. М., 1957.

[5] Татарский В.И. Распространение волн в турбулентной атмосфере. М., 1968.

[6] Герко А.И. Некоторые теоремы существования устойчивых по Лагранжу, устойчивых по Пуассону, рекуррентных в смысле Биркгофа, дистальных и почти периодических в смысле Бора решений многомерных дифференциальных уравнений // Изв. АН ССР Молдова. Матем. 1991. № 1. C. 7-13.

[7] Герко А.И. Согласованные решения многомерных дифференциальных уравнений // Изв. АН РМ. Матем. 1993. № 1. С. 85-90.

[8] Герко А. И. Согласованные решения линейных многомерных дифференциальных уравнений // Изв. АН РМ. Матем. 1994. № 1. С. 42-47.

[9] Шербаков Б.А. Многомерные динамические системы // Дифференц. уравнения. 1994. T. 30. № 5. C. $739-747$.

[10] Герко А.И. Согласованные решения квазилинейных многомерных дифференциальных уравнений // Изв. АН РМ. Матем. 1995. №1. С. 3-11.

[11] Hâncu V. I. Приводимость линейных вполне интегрируемых систем с квазипериодическими коэффициентами // Изв. АН РМ. Матем. 1996. № 1. С. 24-30.

[12] Glavan V.A., Hâncu V. I. Partial recurrent solutions of completely integrable systems // Изв. АН РМ. Матем. 1998. №1 (26). С. 103-112.

[13] Бронштейн И.У., Черний В.Ф. О расширениях динамических систем с равномерно асимптотически устойчивыми точками // Диффференц. уравнения. 1974. Т. 10. № 7 . C. $1225-1230$.

[14] Левитан Б. М., ЖКиков В. В. Почти периодические функции и дифференциальные уравнения. М.: Изд-во МГУ, 1978.

[15] Бронштейн И.У. Расширения минимальных групп преобразований. Кишинев: Штиинца, 1975.

[16] Bronstein I. U. Extensions of Minimal Transformation Groups: Sijthoff \& Noordhoff International Publishers, 1979.

[17] Герко А.И. Рекурсивность, минимальные множествава и устойчивость по Ляпунову в полугруппах преобразований // Матем. исследования (Кишинев). 1972. №24. С. 62-80.

[18] Герко А. И. Об асимптотических относительно расширения свойствах точек топологических полугрупп преобразований // Изв. АН РМ. Матем. 1995. № 2-3. С. 66-75.

[19] Шефер Х. Топологические векторные пространства. М.: Мир, 1971.

[20] Келли Дж. Л. Общая топология. М.: Наука, 1968.

[21] Герко А.И. Poisson stability of mappings with respect to a semigroup // Изв. АН РМ. Матем. 1998. №1. С. 95-102.

[22] Левитан Б. М. Почти периодические функции. М., 1953.

[23] Шербаков Б. А. Устойчивость по Пуассону движений динамических систем и решений дифференциальных уравнений. Кишинев: Штиинца, 1985.

[24] Auslander L., Hahn F. Real functions coming from flows and concepts of almost periodicity // Trans. Amer. Math. Soc. 1963. V. 106. P. 415-426.

[25] Knapp A. W. Distal functions on groups // Trans. Amer. Math. Soc. 1967. V. 128. P. 1-40.

[26] Бронштейн И.У. К теории дистальных минимальных множеств и дистальных функций // Докл. АН СССР. 1967. Т. 172. № 2. С. 255-257.

[27] Чебан Д. Н. Асимптотическая устойчивость по Пуассону решений операторных уравнений // Дифференц. уравнения. 1977. Т. 3. № 8. С. 1411-1417. 